\section{CONDITIONED REFLEXES}

Lectures on Conditioned Reflexes

Vol. 2 : Conditioned Reflexes and Psychiatry. By Prof. Ivan Petrovitch Pavlov. Translated and edited by Dr. W. Horsley Gantt. Pp. $199+7$ plates. (London : Lawrence and Wishart, Ltd., 1941.) 8s. $6 d$. net.

NE is often grateful for the existence of general surveys which are written for international congresses or commemorative volumes. They provide excellent opportunities of summarizing a subject in a form which gracefully supplies entertainment and instruction. It is very doubtful, however, if there is any justification for the collection and republication of the general reviews written by a single author. Inevitably they cover large areas of the subject, and inevitably they overlap. Brief accounts of the same illustrative experiment reappear time after time, until one longs for tables of data and experimental protocols. Of the fifteen chapters in this volume, eleven consist of general articles. The translator has carried out extensive condensation and excision, but far too much that is redundant remains.

The last chapter is by far the best. It was written in 1935, the year before Pavlov's death, and in it he has summarized in twenty pages the whole of his work on conditioned reflexes. It is the most complete and orderly presentation in the book. The other outstanding chapter appeared in the Psychological Review in 1932, translated by Dr. R. S. Lyman. Its style is clear and vigorous, and the subject-matter is scientific polemic at its best. Pavlov defends himself against Guthrie and Lashley by a masterly restatement of his own position, and his criticism of Lashley is fair and penetrating. His comments on experiments on maze-running exemplify his approach to a problem and his instinct for profitable analysis.

The remainder of the book is disappointing. The translation is obscure and clumsy, and there are no references. The introduction contains a biographical sketch of Pavlov's later years, and a section entitled "A Critical Evaluation of Pavlov's Work". This consists of a hotchpotch of the considered and unconsidered opinions of physiologists and psychologists, and a remark addressed to the author by Bernard Shaw at Lady Astor's.

Since 1927, Pavlov and his school seem to have turned their attention chiefly to the study of the disturbances of behaviour, which he calls experimental neuroses. The methods used include the application of very strong stimuli, the protracted use of inhibitory stimuli, the presentation of positive and inhibitory stimuli in quick succession, and castration. On the basis of their behaviour in these conditions, Pavlov divides his dogs into choleric, sanguine, phlegmatic and melancholic types. This classification depends on incompletely defined concepts of 'strong' and 'weak' nervous systems, but it is claimed that it is of use in the production of neuroses at will, and in their cure by appropriate treatment.

When he was more than seventy, Pavlov plunged into the study of human psychiatry. He began to attend psychiatric clinics, and re-opened the writings of the clinical psychiatrists. Naturally, he began to interpret these new phenomena in more familiar terms. In these lectures, analogies abound between human neurasthenia and experimental neuroses, between schizophrenia and chronic hypnosis in dogs. Paranoia and obsessional neuroses are said to be manifestations of pathological inertia of points of the cortex. The classification of men into artists and thinkers, and the conception of words as conditioned stimuli of a second order are further examples of Pavlov's passion for simple hypotheses with which to try to reduce chaos to order.

It is a pity that Pavlov did not begin by trying to verify in man his conclusions from animal experiment. Instead, he writes as one bringing light to the blind. In 1927, he began the last chapter of "Conditioned Reflexes" with a firm warning against the premature application of "these first steps in elucidating the physiology of the cortex ... to the intricate problems of the higher psychic activities in man, when at the present stage of our work, no detailed application of its results to man is yet permissible". It is difficult to feel that the subsequent experimental work described here has provided the evangel that alone could justify Pavlov's disregard of his own warning. He even supplies evidence himself that his results on the dog are not, in fact, generally applicable. Spealking of the spread of prolonged inhibition over the hemispheres in a dog, he remarks that "this has been shown from the experiments by Voskresensky on monkeys, to be a special property of the dog's nervous system". In spite of this, we find that this spread of inhibition over the cortex is invoked to explain sleep, hypnosis, and the states of hebephrenia and catatonia in man.

The main difficulty in Pavlov's work, the extreme complexity of conditions from which the stimulus had to be isolated, was brilliantly overcome. The second major difficulty, the degree to which experimental conditions interfered with normal cortical function, he at least recognized: "Hypnotization begins when the dog enters the experimental room, sometimes even before he gets on the stand, increasing as the experiment progresses". The insuperable difficulty was that Pavlov was limited to external observation of stimulus and response. $\mathrm{He}$ could trace roughly the anatomical pathways involved, but could know nothing of the intermediate processes. Studies on the whole animal must always be interpreted in the light of analytical experiments, and in the absence of such knowledge it is not surprising that Pavlov has bridged the gap between stimulus and response by a mass of hypotheses. Sometimes, by varying his conditions, he has been able to weed out false hypotheses, but often they are left to buttress one another. Attempts by others to re-interpret his data have not been more successful. Denny Brown's substitution of 'attention' and 'distraction' for Pavlov's 'irradiation' and 'waves of inhibition' seems to depend on personal preference, and the great advances in our knowledge of the behaviour of the spinal cord in the last ten years have made interpretations based on classical cord physiology look a little premature.

With the development of a real physiology of the cortex by Adrian, Bremer and Dusser de Barenne, a surer basis for interpretation is being built up. It is already possible to put some of Pavlov's broader hypotheses to experimental test by electrical methods in the intact animal. Studies of cerebral activity in animals will for long be based on Pavlov's work, and the English-speaking worker will turn first to Anrep's translation of "Conditioned Reflexes". If the present volume induces new students of cortical physiology to turn back to that great work, it will have been of lasting value.

D. WHITTERIDGE. 